DOCTRINA

\title{
La relevancia del derecho antártico frente a los desafíos regionales y el papel primordial de Argentina y Chile en su fortalecimiento
}

\author{
The relevance of Antarctic Law in the face of regional challenges \\ and the primary role of Argentina and Chile in its strengthening
}

\author{
Bruno Arpi \\ Universidad de Tasmania, Australia
}

\begin{abstract}
RESUMEN La comunidad antártica ha desarrollado un sistema jurídico, conceptualizado aquí como derecho antártico, para abordar los problemas particulares de la región. Este régimen jurídico regional ha regulado las actividades humanas en el continente antártico y sus aguas circundantes durante las últimas seis décadas. A pesar de gozar en la actualidad con la gran aceptación de la sociedad internacional, se observa un análisis abrumadoramente crítico al régimen jurídico antártico por parte de algunos académicos, argumentando en favor del establecimiento de un régimen global o universal para la región. Estos planteos, que no son nuevos y se los consideraba superados, tienen relevancia para Estados como Argentina y Chile, que han mantenido una postura uniforme y constante a través de la historia: debe ser la comunidad antártica quien tenga la responsabilidad de regular la región en interés de toda la humanidad. Este trabajo tiene como objetivo, por lo tanto, responder a dichas críticas resaltando la importancia de la naturaleza jurídica regional del derecho antártico ante los desafíos regionales y, al mismo tiempo, enfatizando el papel que han jugado y deben continuar jugando Argentina y Chile en su fortalecimiento.
\end{abstract}

PALABRAS CLAVE Sistema del Tratado Antártico, regionalismo, regímenes jurídicos.

ABSTRACT The Antarctic community has developed a legal system, conceptualized here as Antarctic Law, to address the particular problems of the region. This regional legal regime has regulated human activities on the Antarctic continent and its surrounding waters for the past six decades. Despite Antarctic Law currently enjoying great acceptance by international society, there is an overwhelmingly critical analysis of the Antarctic legal regime by some Antarctic scholars, arguing in favour of the establishment of a global or universal regime for the region. These arguments, which are not new 
and were considered to be outdated, are relevant for States such as Argentina and Chile, who have maintained a uniform and constant position throughout history: it must be the Antarctic community that has the responsibility of regulating the region in interest of all humanity. This paper aims therefore to respond to these critics, highlighting the importance of the regional legal nature of Antarctic Law in the face of regional challenges, and at the same time, emphasizing the role that Argentina and Chile have played and should continue to play in their strengthening.

KEYWORDS Antarctic Treaty System, regionalism, legal regimes.

\section{Introducción}

El derecho internacional cumple funciones fundamentales dentro de la sociedad internacional al proporcionar un orden jurídico para la conducción de las relaciones internacionales. Dentro de la amplia libertad de disposición que el derecho internacional universal y general otorga a los Estados (sus principales sujetos), estos pueden constituir regímenes jurídicos regionales en un espacio geográfico continuo para abordar de manera más efectiva problemas particulares o específicos de la región. Los regímenes regionales no son una forma de autoexclusión de la sociedad internacional, sino que forman parte integral de ella, y se enmarcan dentro de los principios estructurales del derecho internacional, como la soberanía y la igualdad de todos los Estados y las normas imperativas (ius cogens) (Remiro Brotóns y otros, 2010: 63).

A partir de la adopción del Tratado Antártico y la posterior evolución del Sistema del Tratado Antártico, la región antártica posee un estatus jurídico y político especial. ${ }^{1}$ Los Estados activos en la región han desarrollado un régimen jurídico regional para abordar los desafíos y problemas del continente antártico y sus aguas circundantes. Durante las últimas seis décadas, este régimen jurídico, conceptualizado aquí como derecho antártico, ha sido la base del orden jurídico y político de la comunidad antártica (el conjunto de Estados y otros sujetos del derecho internacional con intereses comunes en la región antártica). ${ }^{2}$ En la actualidad, el cambio climático y el aumento

1. Esta idea aparece en el Preámbulo de la Convención para la Reglamentación de las Actividades sobre Recursos Minerales Antárticos de 1988 (CRARMA), el Preámbulo del el Protocolo sobre Protección Ambiental del Tratado Antártico de 1991 (Protocolo de Madrid) y en el Acuerdo de Sede de la Secretaría del Tratado Antártico.

2. Esta comunidad, conformada por las partes de los distintos instrumentos que forman el Sistema del Tratado Antártico, está presente en las Reuniones Consultivas del Tratado Antártico (RCTA), las reuniones anuales del Comité para la Protección del Medio (CPA), y las reuniones anuales de la Comisión y el Comité Científico para la Conservación de los Recursos Vivos Marinos Antárticos (CCRVMA y CC-CRVMA, respectivamente). Observadores, como el Comité Científico de Investigaciones Antárticas (SCAR, por sus siglas en inglés), el Consejo de Administradores de Programas Antárticos Nacionales 
del acceso humano a la región plantean nuevos desafíos que probablemente requerirán un mayor desarrollo jurídico e institucional del régimen jurídico antártico.

Dentro de la comunidad antártica, Argentina y Chile han jugado un papel clave en la creación y posterior desarrollo del derecho antártico. Estos dos Estados sudamericanos son signatarios originales del Tratado Antártico y, junto con Australia, Francia, Nueva Zelanda, Noruega y el Reino Unido, son Estados con reivindicaciones territoriales en el continente. Argentina y Chile poseen una larga trayectoria de cooperación antártica y, a pesar de que sus reivindicaciones territoriales se encuentran parcialmente superpuestas, ambos Estados se reconocen mutuamente los derechos soberanos en la Antártica, argumentando que solo resta negociar la delimitación de sus fronteras entre sus respectivos territorios. ${ }^{3}$ No obstante los reiterados intentos de «internacionalización» de la Antártica que se dieron en las décadas de 1940 y 1950, y más tarde en los años ochenta, Argentina y Chile fueron artífices fundamentales en el desarrollo del régimen jurídico regional para la regulación de la Antártica. Conscientes de sus derechos y obligaciones como Estados con derechos soberanos en el continente, Argentina y Chile han promovido - y defendido- la postura que afirma que debe ser la comunidad antártica quien tenga la responsabilidad de regular la región en interés de la toda humanidad. Este régimen jurídico antártico ha sido capaz de proporcionar un orden internacional exitoso ante los desafíos que ha enfrentado la comunidad antártica en las últimas seis décadas.

En la actualidad, el derecho antártico goza de una gran aceptación por parte de la sociedad internacional. ${ }^{4}$ Sin embargo, a pesar de esta aceptación, es posible observar un análisis abrumadoramente crítico al derecho antártico por parte de ciertos académicos. Diversos autores ponen en duda su legitimidad, continúan catalogando como que emana de un «club exclusivo» o argumentan que, ante los nuevos desafíos que enfrenta la comunidad antártica, es hora de repensar la gobernanza de la región en favor del establecimiento de un régimen global o universal (Chaturvedi, 2012; Collis y Stevens, 2004; Dodds, 2006; Elzinga, 2012; Hemmings, 2010a; Hemmings, Dodds y Roberts, 2017; Howkins, 2010; Mancilla, 2020; Yermakova, 2021). A pesar de que

(COMNAP, por sus siglas en inglés); y expertos como la Coalición Antártica y del Océano Austral (ASOC, por sus siglas en inglés) y la Asociación Internacional de Operadores Turísticos de la Antártica (IAATO, por sus siglas en inglés) también están invitados a estas reuniones.

3. Las primeras negociaciones para resolver la delimitación de sus territorios antárticos comenzaron entre 1906 y 1908 (Hayton, 1956: 586 n. 16).

4. El Tratado Antártico, el instrumento central del régimen jurídico, tiene 54 partes contratantes, incluidas veintinueve Partes Consultivas del Tratado Antártico (PCTA), que tienen derecho a participar en la toma de decisiones en la Reuniones Consultivas del Tratado Antártico (RCTA). Las PCTA incluyen a los cinco miembros permanentes del Consejo de Seguridad de las Naciones Unidas. Todos los continentes están representados en las RCTA, y aproximadamente dos tercios de la población humana mundial vive en un país que es parte del Tratado. 
estos planteos no son nuevos y se los creía superados, especialmente desde que la «cuestión de la Antártica» fue removida de la Agenda de la Asamblea General de la Organización de las Naciones Unidas (AGNU) en 2005 y Malasia, su principal impulsor, se uniera al Tratado Antártico en 2011, la internacionalización de la Antártica continúa teniendo relevancia no solo académica, sino que también práctica, especialmente para Argentina y Chile, dos Estados que han mantenido una postura constante a través de la historia en relación con este tema.

Este artículo tiene como objetivo, por lo tanto, responder a las críticas al derecho antártico y a los argumentos en favor de la internacionalización de la Antártica. El trabajo se propone resaltar la importancia de la naturaleza jurídica regional del derecho antártico ante los nuevos desafíos regionales y, al mismo tiempo, enfatizar el papel que han jugado y deben continuar jugando Argentina y Chile en su fortalecimiento. Para lograr dicho objetivo, este artículo procede de la siguiente manera. En la segunda sección se examina el concepto de derecho antártico y sus principales características. La tercera sección analiza críticamente el desarrollo del derecho antártico desde mediados del siglo XX hasta la actualidad y el rol de Argentina y Chile en dicho proceso. La cuarta sección explora las funciones que tiene el derecho antártico dentro de la sociedad internacional contemporánea. Finalmente, la quinta sección enfatiza la relevancia del derecho antártico para el abordaje de los problemas regionales tanto emergentes como existentes que enfrenta la comunidad antártica a principios del siglo XXI.

\section{El derecho antártico como un régimen jurídico regional sui generis}

El derecho antártico puede ser entendido como el sistema de normas y principios jurídicos adoptados por la comunidad antártica para regular la región antártica. ${ }^{5}$ Esta definición requiere algunas aclaraciones adicionales.

5. Esta definición refleja el carácter internacional del sistema; sin embargo, el derecho antártico también comprende una serie de normas nacionales de diferentes Estados. Desde principios del siglo XIX, los Estados - en particular, los Estados reclamantes - han promulgado diferentes normas relativas a la Antártica. El presente trabajo se centra en lo que puede denominarse «derecho internacional antártico». No existe una definición bien aceptada de lo que constituye «la Antártica» o la «región antártica». En este trabajo utilizamos el área político-geográfica adoptada en los tratados que constituyen el régimen jurídico antártico. De conformidad con el artículo VI del Tratado Antártico, las disposiciones se aplicarán «a la región situada al sur de los $60^{\circ}$ de latitud sur, incluidas todas las barreras de hielo»; pero nada del Tratado Antártico «perjudicará o afectará en modo alguno los derechos o el ejercicio de los derechos de cualquier Estado conforme al derecho internacional en lo relativo a la alta mar dentro de esa región». El área de la Convención CRVMA es más amplia. De conformidad con su artículo I.1, la Convención «se aplica a los recursos vivos marinos antárticos de la zona situada al sur de los $60^{\circ}$ de latitud sur y a los recursos vivos marinos antárticos de la zona comprendida entre dicha latitud y la Convergencia Antártica que forman parte del ecosistema marino antártico». 
El derecho antártico no es simplemente un conjunto de normas inconexas, es un sistema de normas y principios jurídicos que se caracteriza por su «integridad, coherencia y unidad» (Combacau, 1986). ${ }^{6}$ El derecho antártico surge principalmente de fuentes convencionales reconocidas por el derecho internacional. ${ }^{7}$ A su vez, dada la amplia representación de la sociedad internacional en el Tratado Antártico y en ausencia de cualquier objeción formal al mismo, al menos algunas de sus disposiciones parecían formar parte del derecho internacional consuetudinario, en particular, el uso de la Antártica con fines pacíficos e investigación científica, como se consagra en el preámbulo y los artículos I y II del Tratado Antártico (Bush, 1982: 51; Jacobsson, 1998: 410).

Como se expresa en todos los instrumentos internacionales adoptados por la comunidad antártica, el derecho antártico busca regir la región en interés de toda la humanidad. ${ }^{8}$ Sobre la base de que los objetivos del régimen jurídico antártico son de interés para la humanidad en su conjunto, y la legitimidad del artículo X del Tratado Antártico, varios autores argumentan que el derecho antártico no solo crea una obligación legal inter partes, sino que también genera ciertas obligaciones erga omnes en relación con la Antártica, como las que están relacionadas con su estado desmilitarizado, la libertad de investigación científica y la protección del medioambiente antártico (Dupuy, 1960: 122; Guyer, 1973: 223-226; Guyer, 1983: 278-279; Jacobsson, 1998: 410; Wolfrum, 1995: 87). ${ }^{9}$ Otros autores han ido más allá y han llegado a la conclusión de que el derecho antártico es un «régimen objetivo» que protege los intereses

6. La idea de un «sistema antártico» fue utilizada por primera vez por el exdiplomático argentino Roberto Guyer cuando pronunció su conferencia en la Academia de Derecho Internacional de La Haya en 1973 (Guyer, 1973). Si entendemos que un régimen jurídico es un sistema de normas que gobiernan un tipo de actividad o un objeto espacial de derecho internacional que ha alcanzado un nivel adecuado de coherencia (Stokke y Vidas, 1996: 13-14), podríamos concluir que el derecho antártico también podría denominarse «régimen jurídico antártico», ya que ambos términos derecho antártico y régimen jurídico antártico se refieren a la misma idea de un sistema coherente.

7. El derecho antártico consiste en el Tratado Antártico de 1959, la Convención para la Conservación de las Focas Antárticas de 1972 (CCFA), la Convención sobre la Conservación de los Recursos Vivos Marinos Antárticos de 1980 (Convención CRVMA) y el Protocolo sobre Protección Ambiental del Tratado Antártico de 1991 (Protocolo de Madrid). Estos tratados, junto con las medidas vigentes en virtud de ellos, constituyen el derecho antártico. En este sentido, esta concepción del derecho antártico vuelve a enfatizar la naturaleza jurídica del término sistema del Tratado Antártico de acuerdo con el artículo 1.e del Protocolo de Madrid de 1991.

8. Esta idea se incluyó en la invitación que Estados Unidos envió a los otros once Estados involucrados en el Año Geofísico Internacional 1957-1958 para participar en la Conferencia de Washington de 1958.

9. Artículo X del Tratado Antártico: «Cada una de las partes contratantes se compromete a hacer los esfuerzos apropiados, compatibles con la Carta de las Naciones Unidas, con el fin de que nadie lleve a cabo en la Antártica ninguna actividad contraria a los propósitos y principios del presente Tratado». 
de todos los Estados, independientemente de si son parte del Tratado Antártico (Beltramino, 1987: 3; Waldock, 1964: 184-185)..$^{10}$

En resumen, el derecho antártico es un ordenamiento jurídico que surge de la comunidad antártica y emana de una serie de tratados internacionales - de los cuales varias disposiciones forman parte del derecho internacional consuetudinario- $y$ medidas vigentes adoptadas bajo estos tratados para ordenar el continente antártico y sus aguas circundantes. El derecho antártico proporciona un orden internacional en la región antártica en interés de toda la humanidad, al proporcionar derechos y obligaciones tanto para la comunidad antártica como para terceros Estados. A partir de estas características, queda claro que el derecho antártico es un derecho internacional regional sui generis.

\section{El desarrollo del derecho antártico por parte de la comunidad antártica y el papel de Argentina y Chile}

El derecho antártico no es estático, sino un sistema dinámico. Este nace y se va desarrollando para responder a distintos desafíos que surgen dentro de la comunidad antártica. La adopción del Tratado Antártico es una respuesta de la comunidad a problemas regionales específicos. En la década de 1950 existía un riesgo significativo de que la Antártica se convirtiera en un escenario de discordia o conflicto internacional (van der Essen, 1997: 17). Había una creciente preocupación de que la región antártica se convirtiera en un escenario de la Guerra Fría entre los Estados Unidos y la Unión Soviética, los cuales tenían importantes intereses en el continente. A su vez, las tensiones se intensificaron debido a las diferentes actitudes hacia los reclamos de soberanía no resueltos. Un potencial conflicto militar entre Argentina, Chile y el Reino Unido (tres aliados de Estados Unidos) en la península Antártica por sus reclamaciones superpuestas era preocupante para Estados Unidos, ya que temía que la Unión Soviética se aprovechara de la situación. ${ }^{11} \mathrm{Al}$ mismo tiempo, surgieron incerti-

10. El régimen objetivo es una categoría de tratado que causa efectos generales y contiene obligaciones erga omnes con respecto a los Estados que no son partes en ellos (Fernández de Casadevante Romani, 2010). La idea del derecho antártico como un régimen objetivo fue rechazada por otros autores (Auburn, 1982: 117; Simma, 1986: 189-209).

11. En la década de 1940, Argentina, Chile y el Reino Unido enviaron expediciones militares a la Antártica y ejecutaron operaciones militares allí entre 1943 y 1948, hasta la adopción de un acuerdo tripartito que estipulaba no enviar buques de guerra al sur del paralelo $60^{\circ}$ para la próxima temporada de verano de 1948. Este acuerdo se renovó cada año hasta la entrada en vigor el Tratado Antártico. Sin embargo, este acuerdo no evitó conflictos entre estos Estados, como el incidente en la bahía Esperanza de 1952 y el incidente de la isla Decepción de 1953. El Reino Unido intentó llevar a Argentina y Chile ante la Corte Internacional de Justicia en 1955, pero los Estados sudamericanos rechazaron la competencia de la Corte. 
dumbres antes del final del Año Geofísico Internacional (AGI) de 1957-1958, cuando varios Estados - incluida la Unión Soviética- decidieron mantener algunas de las estaciones que habían establecido durante este esfuerzo científico. La comunidad antártica estaba al borde de una situación que fácilmente podría haber resultado en una confrontación política, o incluso militar, entre Estados (Guyer, 1983: 270).

En esta época también se dan los primeros intentos de «internacionalizar» la Antártica. Por ejemplo, la Liga Internacional de las Mujeres por la Paz y la Libertad propuso en 1947 la creación de una Comisión de Mandato bajo el Consejo de Administración Fiduciaria de Naciones Unidas. Durante 1948, Estados Unidos primero propuso incorporar la Antártica en el Consejo de Administración Fiduciaria, y luego la creación de un «condominio». Nueva Zelanda también propuso poner la Antártica bajo el control del Consejo de Administración Fiduciaria en 1956. E India trató de plantear la «cuestión de la Antártica» en la agenda de la AGNU en 1956 y 1958. Sin embargo, ninguna de estas propuestas fue aceptada por varios Estados activos en el continente, especialmente Argentina y Chile. Por ejemplo, las propuestas estadounidenses de 1948 fueron categóricamente rechazadas por Argentina, y Chile respondió con una contrapropuesta, que luego se conoció como la «Declaración Escudero» y en la que se encuentra basado el actual artículo IV del Tratado Antártico. Lo mismo sucedió en 1956, cuando la fuerte oposición diplomática de Argentina y Chile hicieron que India retirara su propuesta de colocar la «cuestión de la Antártica» en la agenda de la AGNU.

El riesgo de la participación soviética en la Antártica aceleró los esfuerzos de Estados Unidos para encontrar una solución a la situación compleja de la región a finales de la década de 1950. Así, el 2 de mayo de 1958, Estados Unidos envió una invitación a los otros once Estados participantes en el AGI de 1957-1958 para asistir a una conferencia en la que se promovía que los intereses de la humanidad serían mejor servidos, en consonancia con los altos ideales de la Carta de la Organización de las Naciones Unidas (ONU), si los países que tienen un interés directo en la Antártica se unían en la celebración de un tratado para permitir la libertad de investigación científica y la continuación de la cooperación científica internacional del AGI, garantizar que la Antártica se utilice únicamente con fines pacíficos y cualquier otro fin pacífico que no sea incompatible con la Carta de la ONU. Dado que ningún Estado fue excluido de la participación en el AGI de 1957-1958, este proporcionó criterios objetivos para determinar un interés real en la región. Por ejemplo, Alemania, España y Suecia fueron invitados a participar en el AGI de 1957-58, pero rechazaron estas invitaciones por distintas razones (Guyer, 1973: 177).

La conferencia formal tuvo lugar en Washington entre octubre y diciembre de 1959, y fue preparada mediante consultas informales por representantes diplomáticos de los doce países desde mediados de 1958. El Tratado Antártico se firmó el 1 de diciembre de 1959, con los dos objetivos principales de garantizar el uso pacífico y la 
libertad de la investigación científica en la Antártica. Argentina y Chile desempeñaron un papel clave en la limitación del alcance del Tratado Antártico y en dejar en claro que la Antártica debía estar regulada bajo un régimen regional. El 15 de octubre de 1959, durante el primer día de la Conferencia del Tratado Antártico celebrada en Washington, el embajador de Argentina, Adolfo Scilingo, afirmó que la Conferencia se convocó principalmente para prever el «uso pacífico exclusivo de la Antártica» y el desarrollo de la «cooperación científica» en la región, y enfatizó que «no ha sido convocada para afectar o desconocer derechos». El delegado de Chile coincidió con Argentina en el propósito «limitado» de la Conferencia y reafirmó que Chile «no puede aceptar ninguna fórmula que implique la internacionalización de su territorio antártico» (Hanessian, 1960: 464).

La adopción del Tratado fue posible gracias a su artículo IV, también conocido como modus vivendi, que crea el marco jurídico necesario para la protección de diversas posiciones de los Estados en relación con la soberanía territorial en continente. Si bien el Tratado Antártico no ha resuelto este problema, ha eliminado la necesidad de que las partes reafirmen repetidamente sus puntos de vista respectivos sobre cuestiones de soberanía. El artículo IV — cuya disposición se basa en una propuesta del profesor chileno Julio Escudero en 1948- ha proporcionado una «fórmula ingeniosa» por la cual los miembros de la comunidad antártica «acuerdan estar en desacuerdo» sobre el estatus legal de los reclamos territoriales en la Antártica, mientras que al mismo tiempo proporciona un marco que facilita la cooperación entre ellos (Triggs, 1987: 89). Este artículo ha salvaguardado los diferentes intereses de los países al preservar el statu quo en relación a las reivindicaciones territoriales en la Antártica y los derechos de los Estados sobre dicho territorio.

Los Estados con interés directo son las partes consultivas del Tratado Antártico (PCTA). Estos Estados tienen una responsabilidad especial hacia la sociedad internacional para asegurar que todas las actividades en la Antártica sean consistentes con los propósitos y principios del Tratado Antártico. ${ }^{12}$ Los doce signatarios originales del Tratado Antártico tienen ipso facto e in perpetuum, mientras el Tratado Antártico esté vigente, estatus de parte consultiva. Para que los Estados adherentes obtengan este estatus deben demostrar «interés en la Antártica mediante la realización de una actividad de investigación científica sustancial allí, como el establecimiento de una estación científica o el envío de una expedición científica» (artículo IX.2 del Tratado

12. Esta idea apareció en la RCTA, Recomendación 9-1 de 1977, y ha sido reiterada en varias oportunidades, como en el preámbulo del Protocolo de Madrid y en el Acuerdo de Sede de la Secretaría del Tratado Antártico. Ya en la Conferencia Antártica de diciembre de 1959, el líder de la delegación australiana, lord Casey, dijo que «los doce países reunidos en esta Conferencia son los que tienen contrato directo y responsabilidades en la zona y es bastante adecuado que tengan la tarea de elaborar un Tratado para la zona» (véase Hayton, 1960: 357). 
Antártico). Los Estados que mejor comprenden los desafíos de la Antártica son los que emprenden acciones concretas y enfrentan los desafíos de la dura e inhóspita región. Por tanto, es comprensible que esos Estados tengan la responsabilidad primordial de desarrollar el marco jurídico e institucional del régimen jurídico antártico. La ciencia es la moneda de cambio del reconocimiento como parte consultiva, ya que se basa en la influencia decisiva de los científicos en la adopción del Tratado.

Tras la entrada en vigor del Tratado en 1961, la comunidad antártica se vio ante la necesidad de dar respuesta a desafíos no abordados por dicho tratado, en particular, la protección del medioambiente antártico. Así, la comunidad, de conformidad con el artículo IX.1.f del Tratado, ${ }^{13}$ desarrolla una serie de instrumentos internacionales para proteger el medioambiente antártico. Cabe destacar que todo el desarrollo del régimen jurídico antártico se ha logrado por consenso. Aunque no están codificadas ni definidas dentro de los instrumentos primarios del régimen, las decisiones en las diferentes instituciones que regulan la región antártica se toman por consenso (Jackson, 2018: 246). Consenso no significa unanimidad, sino más bien la ausencia de objeciones formales (Watts, 1992: 56). A pesar de que alcanzar el consenso necesario para adoptar una medida puede ser un proceso lento, este mecanismo de toma de decisiones ha contribuido a un alto grado de cumplimiento. El consenso es el núcleo del régimen jurídico antártico y refleja el espíritu de cooperación del Tratado. La gobernanza antártica no ha estado libre de tensiones internas y externas. Sin embargo, el régimen jurídico de la Antártica se basa en la capacidad de las partes para manejar estas tensiones de manera pacífica, buscando un consenso general.

El primer instrumento importante que se adoptó fueron las Medidas Convenidas para la Protección de la Fauna y la Flora Antárticas de 1964. Luego, las partes consultivas reconocieron la preocupación general sobre la vulnerabilidad de las focas antárticas a la explotación comercial, por lo que adoptaron la Convención sobre la Conservación de las Focas Antárticas (CCFA) de 1972, la que entró en vigor en 1978. En 1980, las negociaciones posteriores dieron como resultado la adopción de la Convención sobre la Conservación de los Recursos Vivos Marinos Antárticos (Convención CRVMA), que entró en vigor en 1982. Luego, la atención se centró en la posible explotación de los recursos minerales. Ante este debate por parte la comunidad antártica, nuevos planteos para establecer un régimen universal para la Antártica aparecieron en el marco de las Naciones Unidas. Sri Lanka hizo un primer intento de llevar el debate de la regulación de la región ante el Consejo Económico y Social y la AGNU en 1975. Luego, desde 1983 hasta 2005, se incluyó en la agenda de la AGNU la «cuestión de la Antártica», una propuesta liderada por Malasia para la «internacio-

13. Este inciso, que fue una propuesta chilena, le otorga a las PCTA la facultad de recomendar a sus gobiernos medidas para promover los principios y objetivos del Tratado, incluidas medidas relativas, entre otras cosas, a la preservación y conservación de los recursos vivos en la Antártica. 
nalización» del continente bajo el mandato de las Naciones Unidas. Sin embargo, la comunidad antártica - con una activa participación de Argentina y Chile- disipó cualquier desafío, fortaleciendo el régimen regional y manteniendo su posición como responsable de regular la Antártica en interés de toda la humanidad. ${ }^{14}$

Debido a la preocupación que surgía de la posible exploración y explotación de recursos minerales en el Área del Tratado Antártico, en 1988 se adoptó la Convención sobre la Reglamentación de las Actividades sobre Recursos Minerales Antárticos (CRAMRA). Sin embargo, dada la fuerte presión de grupos ambientalistas y la no ratificación por parte de Australia y Francia, esta Convención no entró en vigor. En su lugar, la comunidad antártica adoptó el Protocolo de Madrid en 1991, el que entró en vigor en 1998. Este innovador instrumento internacional proporciona reglas ambientales integrales para la región y entre otras cosas estableció el Comité de Protección Ambiental (CPA) para brindar asesoramiento y formular recomendaciones a las partes en relación con la implementación de este Protocolo, y prohibió cualquier actividad relacionada con los recursos minerales, salvo la investigación científica.

Este período también experimentó un aumento en el número de partes del Tratado Antártico. A pesar de las críticas mencionadas a la comunidad antártica en la AGNU, la década de 1970 y sobre todo la de 1980 se caracterizaron por una creciente adhesión de Estados al Tratado Antártico. Brasil ratificó el Tratado en 1975 y obtuvo el estatus consultivo en 1983, Alemania en 1979 (estatus consultivo en 1981), India en 1983 (estatus consultivo en 1983) y China en 1983 (estatus consultivo en 1985). No es sorprendente que la amplia aceptación del sistema del Tratado Antártico dentro de la sociedad internacional haya significado que Estados que en el pasado habían sido críticos - como India, Brasil y Malasia - ahora fuesen parte del Tratado Antártico. Esto refleja que comunidad antártica no es un club «exclusivo» $\mathrm{o}$ «aristocrático». El Tratado Antártico, así como también sus instrumentos internacionales separados asociados en vigor, son tratados multilaterales que están abiertos a la adhesión de cualquier Estado (artículo XIII del Tratado Antártico, artículo 12 de la CCFA, artículo XXIV de la Convención CRVMA, y artículo 22 del Protocolo de Madrid). También es importante de mención que en 2005 la Secretaría del Tratado Antártico abrió en

14. Argentina actuó tras bambalinas en otros foros internacionales, como los Encuentros Cumbre de No Alineados. En 1982, en la AGNU, el primer ministro de Malasia, Mahathir bin Mohamad, declaró que la Antártica «pertenecía a la comunidad internacional». En la siguiente Cumbre del Movimiento de Países No Alineados, Mahathir abogó por que la región fuese considerada como un «patrimonio común de la humanidad». Sin embargo, la Declaración Económica emitida al final de la Cumbre redujo las demandas de Mahathir, instando a la ONU a «emprender un estudio integral sobre la Antártica» mientras tomaba nota, no criticaba, el Tratado Antártico. Dicho texto establece que «la Antártica se utilizará en beneficio de toda la humanidad» (en lugar de «patrimonio común de la humanidad»). Dado que todos los textos de las Cumbre del Movimiento de Países No Alineados se adoptan por consenso, esta fue la única forma de lograr que Argentina aceptara el texto (Beck, 1984: 139). 
Buenos Aires para ayudar a la RCTA y al CPA en el desempeño de sus funciones, con el objetivo de fortalecer el sistema del Tratado Antártico y asegurar que todas las actividades en la Antártica sean consistentes con los propósitos y principios del Tratado y el Protocolo de Madrid.

En resumen, durante las últimas seis décadas la naturaleza dinámica del derecho antártico ha sido demostrada por una ampliación significativa del alcance geográfico, institucional y material del régimen jurídico antártico. El alcance geográfico del área de la Convención CRVMA se extiende más allá del área del Tratado Antártico (hasta la Convergencia Antártica) para abordar la conservación de los recursos marinos antárticos. Se establecieron nuevas instituciones (por ejemplo, la Comisión CRVMA) para hacer frente a los desafíos regionales emergentes, como la conservación de la vida marina antártica. Los actores involucrados en el desarrollo del derecho antártico también han aumentado a lo largo de los años. Si bien la adhesión al Tratado solo está abierta para los Estados (artículo XIII), la Convención CRVMA está abierta a los Estados y las organizaciones regionales de integración económica, cuando cumplan con los requisitos establecidos en su artículo XXIX. En virtud de esta disposición, la Comunidad Europea (hoy Unión Europea) adhirió a la Convención CRVMA en 1982. En cuanto al alcance material, el desarrollo es aún más significativo. Si bien el Tratado Antártico puede considerarse como un acuerdo regional para tratar asuntos relacionados con el mantenimiento de la paz y la seguridad internacionales de acuerdo con el artículo 52 de la Carta de la ONU (Hayton, 1960: 366), con el desarrollo del sistema del Tratado Antártico, el régimen que gobierna la Antártica va más allá de ser solo un régimen de seguridad. Como establece el artículo 2 del Protocolo de Madrid, «la Antártica es una reserva natural dedicada a la paz y la ciencia». La comunidad antártica afirma que «es en interés de toda la humanidad que la Antártica continúe utilizándose siempre exclusivamente para fines pacíficos y que no llegue a ser escenario u objeto de discordia internacional» (preámbulo del Tratado Antártico), y que «la protección del medioambiente antártico y los ecosistemas dependientes y asociados interesa a la humanidad en su conjunto» (preámbulo del Protocolo de Madrid). ${ }^{15} \mathrm{La}$ evolución de derecho antártico nos permite observar cómo la comunidad antártica ha ido fortaleciendo el régimen jurídico regional para hacer frente a los desafíos particulares de la región y, a su vez, que tanto Argentina como Chile han sido clave en este proceso.

\section{El derecho antártico en la sociedad internacional contemporánea}

El derecho antártico emana de la comunidad antártica y es a partir de las características de esta comunidad que podemos comprender los rasgos y funciones del sistema

15. Para una mejor comprensión del significado y los desafíos que conlleva «el interés común de la humanidad» sobre la región antártica, véase Ferrada (2021). 
jurídico antártico. La comunidad antártica forma parte integral de la sociedad internacional y comparte sus principales características. Original y básicamente, es una comunidad de Estados soberanos e independientes. Los cambios sociales vinculados al proceso de globalización han atraído a una serie de actores, pero ni hay un gobierno sin Estados, ni los Estados han sido superados como unidades básicas de la sociedad internacional. Por tanto, la comunidad antártica tiene las mismas características de la sociedad internacional: es una comunidad horizontal, descentralizada, apenas institucionalizada, con un número limitado de sujetos (Pastor Ridruejo, 2010: 47-64) que, partiendo del principio de igualdad soberana, buscan perseguir su convivencia y, eventualmente, articular la cooperación para satisfacer sus intereses comunes. El derecho antártico es imperfecto, pero al igual que el derecho internacional general, es lo que los Estados quieren que sea.

A nivel internacional, todos los Estados - fuertes y débiles, grandes y pequeñosnecesitan un marco de reglas justas, que cada uno pueda estar seguro de que otros obedecerán ${ }^{16}$ y cuya violación tiene un precio. En la región antártica, los Estados han constituido un régimen jurídico regional para abordar los problemas de la región desde la perspectiva de los Estados que están más conectados con ella y la entienden mejor. Este régimen promueve un cierto orden y previsibilidad necesarios para conducir las relaciones internacionales. El derecho antártico es, como hemos visto en su desarrollo, un ajuste o acomodación de intereses estatales en conflicto. El orden establecido por el derecho antártico, aunque sea imperfecto, permite maximizar la ganancia de la utilidad general de los Estados involucrados y, de hecho, incluso evitar el caos en la red de relaciones bilaterales y multilaterales que esa sociedad abraza (Higgins, 1995: 1).

El derecho antártico promueve la paz y la seguridad en la Antártica. La naturaleza jurídica de las normas establecidas por el derecho antártico restringe el comportamiento de los Estados y contiene con éxito intereses en competencia. El derecho antártico ha evitado o prevenido el surgimiento de nuevos y significativos conflictos internacionales en la región. A su vez, también protege los intereses nacionales en la Antártica. El artículo IV del Tratado Antártico permite que todas las partes cooperen entre sí en la investigación científica de la Antártica sin poner en peligro su posición nacional sobre reclamos territoriales. Ningún Estado se ha visto obligado a aceptar reclamaciones territoriales y, al mismo tiempo, los siete Estados reclamantes no han renunciado a ellas. Ello ha llevado a que el derecho antártico permita la coexistencia y convivencia de Estados con intereses diferentes en la región, facilitando así la cooperación internacional en el continente.

El derecho antártico proporciona un sistema operativo para asegurar los valores

16. Kofi Annan, «Secretary-General's address to the General Assembly», Nueva York, 21 de septiembre de 2004, citado en Rothwell y otros (2010). 
que toda la sociedad internacional desea: seguridad, libertad, conservación y uso sostenible de los recursos, y protección del medioambiente. En este sentido, el derecho antártico contribuye al sano desarrollo de la sociedad internacional hacia una verdadera comunidad internacional, con sujetos que comparten tanto intereses y valores comunes $-\mathrm{y}$ que se conciben a sí mismos sujetos a un conjunto común de reglas en sus relaciones entre sí y compartiendo el funcionamiento de instituciones comunescomo una comunidad de moral, ética e identidades comunes (Bull, 1977; Ellis, 2009).

\section{La relevancia del derecho antártico en la actualidad}

Si bien en las secciones anteriores hemos resaltado las funciones fundamentales que cumple el derecho antártico para la sociedad internacional, existen en la actualidad importantes cuestiones a las que la comunidad antártica deberá prestar atención y responder. Es conocido que el continente antártico y sus aguas circundantes se enfrentan actualmente a importantes desafíos físicos, ecológicos, sociales y económicos (Rayfuse 2007: 196). El cambio climático está produciendo impactos significativos en la región, como el derretimiento de áreas anteriormente cubiertas de hielo. Este nuevo escenario permite un mayor acceso humano a la región y una expansión y mayor diversidad de las actividades antárticas como la pesca, el transporte marítimo, la aviación, el turismo y la bioprospección. Este incremento y diversificación de actividades en la Antártica requerirán un fortalecimiento y un papel más destacado del derecho antártico.

La regulación de la prospección biológica en la región antártica es un claro ejemplo de un desafío actual que enfrenta la comunidad. En los últimos años, es posible notar un crecimiento exponencial de las actividades de prospección biológica en la Antártica (Bowman, 2002). Como han sugerido algunos autores, la bioprospección es uno de los principales desafíos del marco jurídico e institucional antártico (Hemmings, 2010b; Joyner, 2012; McGee y Liu, 2019). Si bien las discusiones sobre la prospección biológica en las RCTA comenzaron a principios de la década de 2000 , las partes consultivas del Tratado Antártico aún no se han puesto de acuerdo sobre cómo gobernar esta actividad. Varios Estados ya han declarado que el régimen jurídico actual no es «adecuado y eficaz» para regular las actividades de prospección biológica en el medioambiente antártico. ${ }^{17} \mathrm{~A}$ nivel mundial, ha habido algunos avances para abordar esta cuestión en las Naciones Unidas en las negociaciones hacia un futuro instrumento internacional jurídicamente vinculante en virtud de la Convención sobre el Derecho del Mar sobre la conservación y el uso sostenible de la diversidad bio-

17. «Report of the informal discussion for the intersessional period of 2017/18 on the draft Code of Conduct for the Exploration and Research in Dome A Area in Antarctica», Reuniones Consultivas del Tratado Antártico, ATCM XLI-CEP XXI, WP 10, presentado por China. 
lógica marina de áreas fuera de la jurisdicción nacional (véanse la Resolución 69/262 y la Resolución 72/249 de la AGNU). No está claro en este momento cómo este instrumento internacional propuesto influiría en el régimen regional que regula el océano Austral (Leary, 2020). Sin embargo, estos avances a nivel mundial no parecen replicarse dentro del régimen jurídico antártico. Durante más de una década, algunos autores han estado pidiendo una respuesta regional a cuestiones como la prospección biológica (Jabour-Green y Nicol, 2003). La comunidad antártica siempre ha estado a la vanguardia del desarrollo de nuevos enfoques y reglas para los problemas que afectan a la región antártica, y no puede quedarse atrás en la regulación de esta actividad. Por lo tanto, es necesario que esta comunidad llegue a un consenso para asumir el liderazgo con respecto a la bioprospección en la Antártica. ${ }^{18}$

A pesar de estos nuevos desafíos a los que se enfrenta la comunidad antártica a principios del siglo XXI, algunos de los desafíos originales de la región no han desaparecido. El desafío de la soberanía territorial, y el mantenimiento y la afirmación continua de esa soberanía, sigue siendo un tema tan vivo como lo fue en la década de 1950 (Rothwell, 1996: 78). Las diferentes actitudes de los Estados hacia la soberanía todavía existen, como se puede observar en las diferentes posiciones de los Estados con reivindicaciones territoriales en sus presentaciones a la Comisión de Límites de la Plataforma Continental, en virtud de la Convención de las Naciones Unidas sobre el Derecho del Mar (véase Hemmings y Stephens, 2010). Los Estados reclamantes nunca han renunciado a sus reivindicaciones territoriales, el artículo IV del Tratado Antártico fue replicado en todos los instrumentos adoptados subsecuentemente por la comunidad antártica. ${ }^{19} \mathrm{El}$ derecho antártico, por lo tanto, sigue desempeñando un papel fundamental en la protección de las respectivas posiciones de los distintos Estados sobre la cuestión de la soberanía territorial en el continente.

\section{Conclusión}

La región antártica goza de un estatus jurídico especial. La comunidad antártica ha desarrollado un sistema de normas jurídicas que ha abordado con éxito los problemas particulares del continente antártico y sus aguas circundantes en interés de toda la humanidad durante las últimas seis décadas. En ese proceso, Argentina y Chile jugaron un papel clave en la creación y posterior desarrollo del régimen regional que

18. «Report...».

19. Por ejemplo, el artículo 1 de la CCFA de 1972 establece que «esta Convención se aplica a los mares al sur de los $60^{\circ}$ de latitud sur, respecto de los cuales las partes contratantes afirman las disposiciones del artículo 4 del Tratado Antártico»; y el artículo IV.1 de la Convención CRVMA indica que, «con respecto al área del Tratado Antártico, todas las partes contratantes, sean o no partes del Tratado Antártico, están obligadas por los artículos IV y VI del Tratado Antártico en sus relaciones entre sí». 
regula la Antártica. Ante los desafíos que ha ido afrontando la comunidad antártica a lo largo de los años, el derecho antártico se ha convertido en un sistema más complejo y más amplio, y ha transformado la región en una reserva natural dedicada a la paz y la ciencia, que considera la protección del medioambiente antártico y los ecosistemas dependientes y asociados en interés de la humanidad en su conjunto.

El derecho antártico cumple funciones importantes para la comunidad antártica, pero también para la sociedad internacional en general. El derecho antártico brinda el orden y la previsibilidad necesarios para llevar a cabo las relaciones internacionales en la región. Este orden imperfecto, entre otras cosas, ha promovido la paz y la seguridad, ha salvaguardado los intereses nacionales, permite la coexistencia de intereses contrapuestos y promueve la cooperación. El derecho antártico asegura valores que toda la sociedad internacional desea: seguridad, libertad, conservación y uso sostenible de los recursos, y protección del medioambiente.

En la actualidad, la comunidad antártica enfrenta desafíos que requerirán un mayor desarrollo jurídico e institucional del derecho antártico. El cambio climático, el mayor acceso humano con el incremento y la mayor diversidad de actividades como la bioprospección en la región antártica, llaman a la comunidad antártica a lograr un consenso en el abordaje normativo del nuevo escenario regional. Al mismo tiempo, el desafío original que enfrentó la comunidad - las diferentes actitudes de los Estados con respecto a la soberanía territorial sobre el continente- sigue siendo un factor importante en la configuración el régimen jurídico antártico.

Ante este escenario, distintos autores han planteado nuevos argumentos en favor del establecimiento de un régimen universal o global para la Antártica. Sin embargo, las funciones que ha cumplido el derecho antártico en la sociedad internacional a través de su historia permiten sostener que los nuevos - como los ya existentes- desafíos que enfrenta la comunidad antártica no hacen más que reforzar la importancia del derecho antártico para la región. La naturaleza regional de este derecho brinda una plataforma única para la comprensión de la interacción humana y el orden de la zona. Es la comunidad antártica la que mejor entiende los problemas específicos de la región y la que cuenta con las mayores herramientas para abordarlos de una manera exitosa, como muestran las últimas seis décadas de gobernanza antártica.

En este éxito mucho ha tenido que ver el papel desempeñado tanto por Argentina como por Chile dentro de la comunidad antártica. Es preciso, por lo tanto, que estos Estados sudamericanos continúen fortaleciendo el régimen jurídico antártico en las décadas venideras, ya que este es importante no solo para la salvaguarda de sus intereses nacionales, sino también para los intereses de toda la humanidad. 


\section{Referencias}

Auburn, Francis (1982). Antarctic law and politics. Londres: C. Hurst \& Co.

Beск, Peter J. (1984). «The United Nations and Antarctica». Polar Record, 22 (137): 137-144. DOI: 10.1017/So032247400005076.

Beltramino, Juan Carlos (1987). «International relations and International Law in Antarctica: A geographical approach». Presentación en el Institut fur Volkerrechts and Internationale Beziehungen, Innsbruck, Austria, 6 de marzo de 1987. Disponible en https://bit.ly/3essqzv.

Bowman, John (2002). "Antarctica a global "hot spot": Biodiversity and biotechnology». Presentación en Looking South: Managing Technology, Opportunities and the Global Environment, 20-21 de noviembre de 2001, Hobart, Tasmania.

BulL, Hedley (1977). The anarchical society: A study of order in world politics. Londres: Macmillan.

Bush, W. M. (1982). Antarctica and International Law: A collection of inter-State and national documents. Londres: Oceana.

Chaturvedi, Sanjay (2012). «India and Antarctica: Towards post-colonial engagement?». En Anne-Marie Brady (editora), The emerging politics of Antarctica (pp. 61-85). Londres: Routledge.

Collis, Christy y Quentin Stevens (2004). «Modern colonialism in Antarctica: The coldest battlefield of the Cold War». Presentación en el 7th Australasian Urban History/Planning History Conference, 12-14 de febrero de 2004. Disponible en https://eprints.qut.edu.au/4605/.

CombacaU, Jean (1986). «Le droit international : Bric-à-brac ou système ?». Disponible en https://bit.ly/zeopYdj.

DodDs, Klaus J. (2006). «Post-colonial Antarctica: An emerging engagement». Polar Record, 42 (1): 59-70. DOI: 10.1017/So032247405004857.

Dupuy, René-Jean (1960). «Le Traité sur l'Antarctique». Annuaire Français de Droit International, 6: 111-132. DOI: 10.3406/afdi.1960.898.

ElLIS, David C. (2009). "On the possibility of "international community"». International Studies Review, 11 (1): 1-26. DOI: 10.1111/j.1468-2486.2008.01822.x.

ELzINGA, Aant (2012). «Rallying around a flag? On the persistent gap in scientific internationalism between word and deed». En Anne-Marie Brady (editora), The emerging politics of Antarctica (pp. 203-229). Londres: Routledge.

Fernández de Casadevante Romani, Carlos (2010). «Objective regime». En Rüdiger Wolfrum (editor), Max Planck Encyclopedia of Public International Law. Max Planck Institute for Comparative Public Law and International Law.

FERRADA, Luis Valentín (2021). «Reflexiones sobre el interés común de la humanidad en el 60. ${ }^{\circ}$ aniversario del Tratado Antártico». Estudios Hemisféricos y Polares, 12 (1): 58-72. Disponible en https://bit.ly/3JmoLOQ. 
Guyer, Roberto (1973). "The Antarctic System». En Collected courses of the Hague Academy of International Law. Volumen 139. Brill. DOI: 10.1163/1875-8096_pplrdc_A9789028604247_03.

—. (1983). «Antarctica's role in international relations». En Francisco Orrego Vicuña (editor), Antarctic resources policy: Scientific, legal and political issues. Cambridge: Cambridge University Press.

Hanessian, John (1960). «The Antarctic Treaty 1959». The International and Comparative Law Quarterly 9 (3): 436-480. DOI: 10.1093/iclqaj/9.3.436.

HaYton, Robert (1956). «The "American" Antarctic». American Journal of International Law, 50 (3): 583-610. DOI: 10.2307/2195508.

-. (1960). «The Antarctic Settlement of 1959». The American Journal of International Law, 54 (2): 349-371. DOI: $10.2307 / 2195252$.

Hemmings, Alan D. (2010a). «After the party: The hollowing of the Antarctic treaty system and the governance of Antarctica». Presentado en Symposium on Antarctic Politics. Disponible en https://hdl.handle.net/10092/17651.

-. (2010b). «Does bioprospecting risk moral hazard for science in the Antarctic Treaty System». Ethics in Science and Environmental Politics, 10 (1): 5-12. DOI: 10.3354/esepoo103.

Hemmings, Alan D., Klaus Dodds y Peder Roberts (2017). «Introduction: The politics of Antarctica». En Klaus Dodds, Alan D. Hemmings y Peder Roberts (editores), Handbook on the politics of Antarctica. Cheltenham: Edward Elgar.

Hemmings, Alan D. y Tim Stephens (2010). «The extended continental shelves of sub-Antarctic Islands: Implications for Antarctic governance». Polar Record, 46 (4): 312-327. DOI: 10.1017/So032247409990532.

Higgins, Rosaly (1995). Problems and process: International Law and how we use it. Oxford: Clarendon.

Howkins, Adrian (2010). «Appropriating space: Antarctic imperialism and the mentality of settler colonialism». En Tracey Banivanua Mar y Penelope Edmonds (editoras), Making settler colonial space (pp. 29-52). Springer. DOI: 10.1057/9780230277946_3.

JABOUR-GrEen, J. y D. Nicol (2003). «Bioprospecting in areas outside national jurisdiction: Antarctica and the Southern Ocean». Melbourne Journal of International Law, 4: 76-111. Disponible en https://eprints.utas.edu.au/2668/.

JACKsOn, Andrew (2018). «Politics, diplomacy, and the creation of Antarctic Consensus». The Yearbook of Polar Law Online, 9 (1): 243-261. DOI: 10.1163/22116427_009010011.

JACOBsson, Marie (1998). The Antarctic Treaty System: Erga omnes or inter partes? Tesis de doctorado, Department of Law, Lund University.

JoYneR, Christopher (2012). «Bioprospecting as a challenge to the Antarctic Treaty». En Alan Hemmings, Donald Rothwell y Karen Scott (editores), Antarctic security 
in the Twenty-First century: Legal and policy perspectives (pp. 197-214). Londres: Taylor \& Francis.

Leary, David (2020). «Research handbook on Polar Law». En Karen N. Scott y David L. VanderZwaag (editores), Bioprospecting at the Poles (pp. 271-291). Cheltenham: Edward Elgar.

Mancilla, Alejandra (2020). «Four principles to justify claims to jurisdiction and to Natural Resources in Antarctica». The Yearbook of Polar Law Online, 11 (1): 170191. DOI: 10.1163/22116427_011010010.

McGeE, Jeffrey y Nengye Liu (2019). «The challenges for Antarctic governance in the early Twenty-first century». Australian Journal of Maritime \& Ocean Affairs, 11 (2): 73-77. DOI: 10.1080/18366503.2019.1634940.

Pastor Ridruejo, José Antonio (2010). Curso de derecho internacional público y organizaciones internacionales. 13. ${ }^{\mathrm{a}}$ ed. Madrid: Tecnos.

RAYFUSE, Rosemary (2007). «Melting moments: The future of polar oceans governance in a warming world». Review of European Community \& International Environmental Law, 16 (2): 196-216. DOI: 10.1111/j.1467-9388.2007.00555.x.

Remiro Brotóns, Antonio, Rosa María Riquelme Cortado, Esperanza Orihuela Calatayud, Javier Díez-Hochleitner Rodríguez y Luis Pérez-Prat Durbán (2010). Derecho internacional: Curso general. Madrid: Tirant lo Blanch.

RothWELl, Donald (1996). The polar regions and the development of international law. Cambridge: Cambridge University Press.

Rothwell, Donald, Stuart Kaye, Afshin Akhtarkhavari y Ruth Davis (2010). International law: Cases and materials with Australian perspectives. Cambridge: Cambridge University Press.

Simma, Bruno (1986). «The Antarctic Treaty as a treaty providing for an objective regime». Cornell International Law Journal, 19 (2): 189-209. Disponible en https:// bit.ly/3FAzcyV.

Stoкке, Olav y Davor Vidas (1996). Governing the Antarctic: The effectiveness and legitimacy of the Antarctic Treaty system. Cambridge: Cambridge University Press.

TRIGgs, Gillian D. (1987). The Antarctic Treaty regime: Law, environment and resources, studies in polar research. Cambridge: Cambridge University Press.

VAN DER Essen, Alfred. (1997). «The origin of the Antarctic System». En Francesco Francioni y Tullio Scovazzi (editores), International Law for Antarctica (pp. 17-29) Leiden: Brill, Nijhoff.

WALDOCK, Humphrey (1964). «Third report on the law of treaties». En Yearbook of the International Law Commission, Vol. II (pp. 5-65). International Law Commission. WATTS, Arthur (1992). International law and the Antarctic treaty system. Cambridge: Grotius.

Wolfrum, Rüdiger (1995). «Possible challenges and the future development of the Antarctic Treaty System». Presentado en Antarctic Horizon: Proceedings of an 
International Symposium on the Future of the Antarctic Treaty System, Ushuaia, Argentina.

Yerma Kova, Yelena (2021). «Legitimacy of the Antarctic Treaty System: Is it time for a reform?». The Polar Journal: 1-18. DOI: 10.1080/2154896X.2021.1977048.

\section{Reconocimiento}

El presente trabajo está basado en la presentación en las Terceras Jornadas Chilenas de Derecho Antártico: «Regionalismo vs. universalismo en la gobernanza antártica: El papel de Argentina y Chile en salvaguardar la importancia del régimen jurídico regional antártico en interés de la humanidad ante la AGNU». Algunos de los aspectos tratados en este artículo fueron abordados en un trabajo anterior del autor. Para más información, véase Bruno Arpi y Jeffrey McGee, «Rediscovering the importance of Antarctic law for the early Twenty-first Century», Australian Journal of International Affairs. Parte de la financiación para esta investigación fue proporcionada por el Australian Research Council Discovery Grant DP190101214: «Geopolitical change and the Antarctic Treaty System».

\section{Sobre el autor}

Bruno Arpi es candidato a doctor en la Facultad de Derecho y en el Instituto de Estudios Marinos y Antárticos de la Universidad de Tasmania, Australia. Maestría en Derecho (LL.M) de la Universidad de Copenhague, Dinamarca. Abogado en la Universidad Nacional de Rosario, Argentina, donde a su vez se despeña como docente de Derecho Internacional Público desde 2017. Su correo electrónico es bruno.arpi@utas. edu.au. (D) https://orcid.org/oooo-0003-4714-7918. 
La Revista Tribuna Internacional busca fomentar la reflexión, el debate, el análisis y la comunicación pluralista y con rigor científico en las áreas del derecho internacional público, derecho internacional privado, relaciones internacionales y derecho internacional de los derechos humanos. Los artículos y ensayos son seleccionados mediante revisión de pares externos a la Facultad de Derecho de la Universidad de Chile. Se reciben trabajos en castellano y en inglés.

EDITOR GENERAL

Luis Valentín Ferrada Walker

SITIO WEB

tribunainternacional.uchile.cl

CORREO ELECTRÓNICO

revistatribuna@derecho.uchile.cl

LICENCIA DE ESTE ARTÍ́CULO

Creative Commons Atribución Compartir Igual 4.o Internacional

La edición de textos, el diseño editorial

y la conversión a formatos electrónicos de este artículo

estuvieron a cargo de Tipográfica

(www.tipografica.io) 